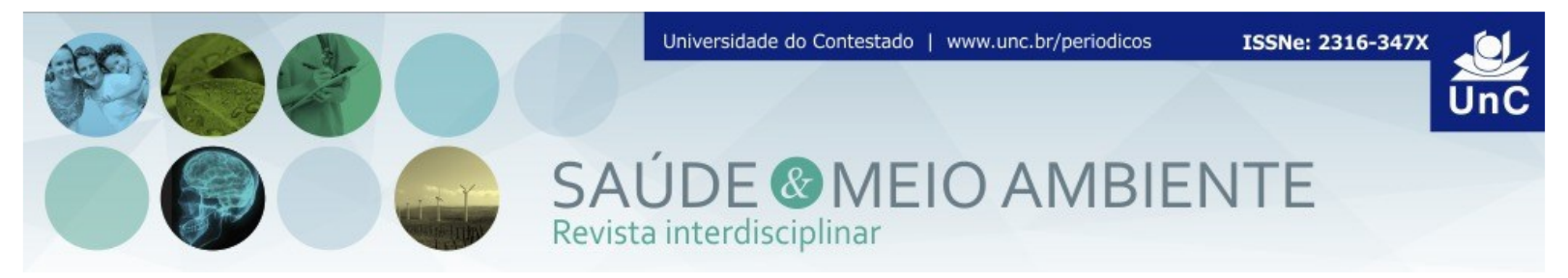

\title{
DESAFIOS DA ENFERMAGEM PARA ATUAÇÃO EM URGÊNCIA E EMERGÊNCIA ${ }^{1}$
}

\author{
Bianca Letícia Sokolski² \\ Fernanda Vandresen ${ }^{3}$ \\ Carlos Otávio Senff ${ }^{4}$
}

\begin{abstract}
RESUMO
A complexidade dos serviços de urgência e emergência relaciona-se às situações críticas dos pacientes ali atendidos, e que precisam de cuidados dirigidos não apenas ao seu estado fisiopatológico, mas também às questões psicossociais, ambientais e familiares. O estudo teve como objetivo identificar os desafios profissionais para a atuação na urgência e emergência na área hospitalar. Trata-se de uma pesquisa exploratória com abordagem qualitativa, voltada a caracterizar a atuação na área de urgência e emergência de profissionais da saúde. O estudo foi desenvolvido em um serviço que presta atendimento de urgência e emergência no Paraná e teve como sujeitos os profissionais que compõem a equipe de enfermagem hospitalar e os pacientes. A coleta dos dados foi realizada com os seguintes instrumentos: entrevista semiestruturada com profissionais de enfermagem; acolhimento dos pacientes após classificação de risco com aplicação de questionário; e análise documental, buscando verificar o histórico de atendimentos e de fatos que possam ser significativos para o estudo a ser desenvolvido. A equipe de enfermagem tem um papel fundamental na área hospitalar, devido contato direto com o paciente para prestar assistência 24 horas por dia. Os desafios para atuação em urgência e emergência hospitalar apontados são a necessidade de atender todos os pacientes de modo rápido e eficiente, realizar a classificação de risco corretamente, administrar o setor de forma organizada, a sobrecarga de trabalho, a superlotação de atendimentos, poucos profissionais em atendimento no setor, entre outros.
\end{abstract}

Palavras-Chave: Equipe de Enfermagem. Emergências. Assistência à Saúde.

\footnotetext{
1 O presente trabalho foi realizado com apoio do Programa de bolsas Universitárias do Estado de Santa Catarina - UNIEDU.

${ }^{2}$ Graduanda de Enfermagem, Universidade do Contestado - UnC. Rua Presidente Nereu Ramos1071 Bairro Jardim Moinho. Mafra. Santa Catarina. Brasil. E-mail: bianca leticia@hotmail.com

3 Professora orientadora. Enfermeira. Mestranda em Desenvolvimento Regional, Docente da Universidade do Contestado Campus Mafra. Rua Presidente Nereu Ramos, 1071 Bairro Jardim Moinho. Mafra. Santa Catarina. Brasil. E-mail: enf fv@yahoo.com.br

${ }^{4}$ Doutor em Administração pela PUCPR (2017). Mestre em Administração pela PUCPR (2012). Especialista em Gestão de Negócios pela FAE/CDE (1998). Graduado em Administração pela FAE Faculdade Católica de Administração e Economia (1995). Diretor do Departamento de Economia e Estatística da Associação Empresarial de Mafra - ACIM. Presidente do Conselho de Desenvolvimento Econômico de Mafra - CODEM. Membro da Diretoria da Associação Beneficente e Cultural NOVA ESPERANÇA, Consultor Independente. Mafra. Santa Catarina. Brasil. E-mail: carlos.senff@gmail.com
} 


\title{
NURSING CHALLENGES FOR EMERGENCY AND EMERGENCY ACTION
}

\begin{abstract}
The complexity of the emergency and emergency services is related to the critical situations of the patients who are cared for, and who need care directed not only to their physiopathological state, but also to psychosocial, environmental and family issues. The objective of this study was to identify the professional challenges for emergency and emergency care in the hospital area. This is an exploratory research with a qualitative approach, aimed at characterizing the performance in the area of emergency and emergency of health professionals. The study was developed in a service that provides emergency and emergency care in Paraná and had as subjects the professionals who make up the hospital nursing team and the patients. Data collection was performed with the following instruments: semi-structured interview with nursing professionals; patient care after risk classification with questionnaire application; and documentary analysis, seeking to verify the history of attendances and of facts that may be significant for the study to be developed. The nursing team plays a key role in the hospital area due to direct contact with the patient to provide care 24 hours a day. The challenges to act in emergency and hospital emergencies are the need to attend all patients quickly and efficiently, perform risk classification correctly, manage the sector in an organized way, work overload, overcrowding of care, few professionals in service in the sector, among others.
\end{abstract}

Keywords: Nursing Team. Emergencies. Delivery of Health Care.

\section{INTRODUÇÃO}

A equipe de enfermagem tem um papel fundamental na área hospitalar, por estar em maior contato com os pacientes e por ser em maior número, prestando cuidados 24 horas por dia. O vínculo do paciente e o hospital são realizados pela enfermagem, criando uma maior responsabilidade de atitudes relacionadas ao cliente, sendo a satisfação do usuário de suma importância[1].

Os pacientes são avaliados e encaminhados à sala de urgência imediatamente ou não, dependendo a situação. Inicialmente é realizado o acolhimento e o cuidado pelo profissional enfermeiro até a chegada do médico.

O enfermeiro deve sempre buscar atualização própria e de toda equipe de trabalho em cursos e evidências para melhorar o atendimento ao cliente. Entretanto, há muitas dificuldades enfrentadas pela equipe, como o desgaste físico, a superlotação de pacientes, a desorganização do ambiente, a pouca quantidade de profissionais. Esses fatores acabam prejudicando o atendimento ${ }^{[2]}$.

Nos serviços de urgência e emergência há muitos casos de pacientes que poderiam ser atendidos em unidades básicas de saúde, pois esses serviços originalmente são destinados a pacientes realmente em urgência e emergência, no 
qual deveria ser mantida a realidade original, evitando tanta demora no atendimento e melhorando a qualidade do mesmo.

Os pacientes preferem consultar em uma unidade de Urgência e Emergência, devido a melhor resolutividade de seus problemas e o fácil acesso às consultas, agilidade na realização de exames, administração de medicamentos, deste modo se torna uma vantagem para os mesmos.

A alta complexidade de uma doença também pode ser um problema, devido à demora em transferência de um paciente de um hospital para o outro, ou de uma área a outra, acaba expondo o paciente a alguns riscos mantendo-o na sala de observação internado, sem as condições que seriam necessárias para o estado do mesmo.

Toda essa superlotação de pacientes resulta em algumas dificuldades vividas pelos profissionais, como o estresse relacionado à sobrecarga de trabalho, ao grande número de pacientes, reclamações, afazeres e a pouca quantidade de profissionais para realização dos atendimentos ${ }^{[3]}$.

Várias dificuldades são enfrentadas nas práticas de saúde, cabe-nos a reflexão de quais os desafios enfrentados pelo enfermeiro na atuação em serviços de urgência e emergência?

Atuar na saúde exige muito conhecimento técnico-científico dos profissionais, pois visa ao bem-estar das pessoas por meio do atendimento de suas necessidades humanas básicas ${ }^{[4]}$.

Embora a tecnologia seja importante para o aprimoramento dos cuidados de enfermagem, não substitui a mão humana, veio sim, para facilitar o desempenho profissional, proporcionando maior disponibilidade de tempo para dedicar ao cuidado do outro, seja ele cliente ou colega da equipe multiprofissional.

Para atuar em serviço de emergência, o profissional precisa ter algumas características, como: calma na abordagem ao paciente, conhecimento e treinamento na área, e na medida em que adquirir experiência, fará um atendimento mais rápido e eficaz ${ }^{[5]}$.

Mediante a necessidade de tornar os serviços de saúde viáveis, por questões econômicas, os hospitais precisam aumentar sua receita e diminuir seus custos. Com isso há a necessidade de refletir sobre os processos, verificando que os profissionais da saúde não devem e não podem mais fazer seus trabalhos sem análise, preparo e conhecimento, demandando a necessidade de profissionais qualificados para assumir cargos de gestão e liderança ${ }^{[4]}$.

O serviço de emergência é considerado um setor muito estressante, ocasionando desgaste físico e mental dos trabalhadores, com efeitos prejudiciais na saúde dos mesmos ${ }^{[6]}$. 
Os fatores estressores podem estar associados não apenas às condições de trabalho e demanda de pacientes, mas devido a baixa remuneração e o sentimento de não valorização do seu trabalho ${ }^{[3]}$.

Existem muitos problemas na infraestrutura da emergência, como por exemplo a sala de observação dos pacientes que transforma-se em sala de internamento sem a estrutura necessária para manter o paciente internado, nem profissionais suficientes para o atendimento, fazendo com que os clientes estejam constantemente em risco[7].

Autores afirmam que a população procura o atendimento de emergência por ser um atendimento rápido, sem nem mesmo precisar dessa urgência, em apenas um dia conseguem marcação de consultas, de exames, e o diagnóstico, sem muita espera[8].

A visão dos pacientes sobre o atendimento de enfermagem nos serviços de urgência e emergência demonstra que para estes esta satisfatório, porém reconhecem que podem ser melhorados ${ }^{[9]}$.

Um dos fatores considerados decisivos para as adversidades na atuação do enfermeiro em urgência e emergência são as inúmeras atribuições exercidas por este profissional, tanto na assistência direta ao paciente, quanto em atividades administrativas e de supervisão de sua equipe ${ }^{[10]}$.

Diante disso, o presente estudo teve como objetivo geral identificar os desafios da enfermagem para a atuação na urgência e emergência na área hospitalar.

\section{MATERIAL E MÉTODOS}

Este estudo se caracteriza com relação aos seus objetivos como exploratório com abordagem quali-quantitativa, realizada por meio de aplicação de questionários com perguntas fechadas de múltipla escolha com escala do tipo Lickert de cinco pontos, voltada a caracterizar a atuação na área de urgência e emergência de profissionais da saúde; e análise documental para verificação dos registros relacionados a satisfação dos usuários.

O estudo foi desenvolvido no segundo semestre de 2018 , em um serviço de saúde que presta atendimento de urgência e emergência no município de Rio Negro/PR, na área hospitalar. A unidade de análise foi escolhida por conveniência de acesso aos respondentes.

O estudo teve como sujeitos os profissionais que compõem a equipe de enfermagem na área de urgência e emergência, e os usuários dos serviços. 
A amostra caracteriza-se como não probabilística, sendo a seleção dos respondentes realizada de maneira intencional em função da possibilidade de acesso e disponibilidade dos respondentes no momento da realização da pesquisa.

Os três questionários com perguntas específicas para cada grupo de respondentes foram adaptados, e foram aplicados para enfermeiros, técnicos de enfermagem e pacientes. Para o grupo de enfermeiros as perguntas foram direcionadas para compreensão da classificação de risco e sobre a assistência prestada. Para os técnicos de enfermagem as perguntas foram compostas para avaliação do atendimento prestado. O questionário dos pacientes foi desenvolvido para compreender a percepção dos usuários quanto a realização da humanização, o acolhimento dos pacientes após classificação de risco e satisfação com relação aos serviços prestados ${ }^{[11,12]}$.

A análise documental foi realizada com a intenção de verificar o histórico de atendimentos e de fatos que possam ser significativos para o estudo desenvolvido.

A seleção dos respondentes foi aleatória, buscando caracterizar a equipe nos serviços de urgência e emergência e também os usuários, avaliando a satisfação dos mesmos. Participaram da pesquisa 08 profissionais da saúde e 54 pacientes. Os profissionais já foram contatados pelos pesquisadores para consulta prévia sobre a participação na pesquisa, onde foram esclarecidos sobre os propósitos do estudo.

A realização da pesquisa foi condicionada a aprovação prévia do Comitê de Ética da Universidade do Contestado, pelo Parecer Consubstanciado nº 2917374.

A tabulação dos dados foi realizada em planilha eletrônica (Microsoft Excel ${ }^{\circledR}$ ), sendo realizadas estatísticas descritivas básicas.

\section{RESULTADOS E DISCUSSÕES}

\subsection{CARACTERIZAÇÃO DA AMOSTRA}

A amostra desse estudo caracterizou pessoas de diversas idades e ambos os sexos. Total de 9 profissionais da equipe de enfermagem, sendo 6 técnicos de enfermagem e 3 enfermeiros.

Foram abordados 54 pacientes e realizadas algumas perguntas sobre a satisfação dos usuários, levando em conta que algumas questões não foram todos que aceitaram responder. 


\subsection{VISÃO DO PACIENTE SOBRE O SERVIÇO DE URGÊNCIA E EMERGÊNCIA}

Para os pacientes, o tempo de espera antes do atendimento foi classificado como $61,1 \%$ (33) bom, 7,4\% (4) péssimo, 3,7\% (2) ruim, 11,1\% (6) regular, 16,6\% (9) Ótimo.

A satisfação inclui a boa comunicação, orientação, confiabilidade, dignidade, respeito ao atendimento rápido, bom acesso ${ }^{[13]}$. De acordo com o Ministério da Saúde, uma das importantes questões que envolvem a classificação de risco é a diminuição do tempo de espera ${ }^{[14]}$.

Outra questão abordada é se o usuário é bem informado sobre os problemas de saúde e o que será realizado. A opinião dos usuários foi $74 \%$ (40) ótimo, 20,3\% (11) bom e 5,5\% (3) regular. Entretanto de acordo com o estudo realizado com amostras de uma unidade de Urgência e Emergência localizada em Fortaleza, de 382 usuários, 50,8\% relataram que não haviam sido bem orientados e reclamaram da qualidade do atendimento, mostrando ainda um grande déficit na comunicação ${ }^{[15]}$.

Referente à humanização do atendimento $90,7 \%$ (49) relataram ser ótimo, $7,4 \%$ (4) bom e 1,8\% (1) regular. Porém no mesmo estudo realizado em Fortaleza, a maioria dos relatos dos usuários envolveu a grande dificuldade do profissional se preocupar com as pessoas e principalmente o péssimo acolhimento recebido[15].

A enfermagem deve estabelecer um bom nível de confiança, esclarecendo tudo de forma clara e objetiva, evitando dificuldades ${ }^{[16]}$.

Ao serem questionados se os profissionais de enfermagem se apresentaram antes da realização dos procedimentos, sob essa adesão caracterizaram da seguinte forma: Péssimo 1,8\%(1) Ruim 1,8\%(1) Regular 27,7\%(15), Bom 31,4\% (17), Ótimo 38,8\% (21).

Em um estudo realizado $70 \%$ dos pacientes também estavam satisfeitos com a apresentação dos profissionais, entretanto relata que é necessária a utilização de crachás ou outras formas de identificações dos profissionais, por direito ao usuário[11].

Sobre a visão geral do atendimento de enfermagem $88,8 \%$ (48) relataram ser ótimo e $11,1 \%$ (6) bom. De acordo com estudos, o profissional de saúde deve estar aberto ao diálogo, criando um vínculo, deste modo favorecendo o atendimento para o pronto atendimento, obtendo a participação do usuário durante o atendimento, para melhor resolutividade ${ }^{[17]}$.

A satisfação do usuário está relacionada tanto ao acesso quanto ao acolhimento recebido, os pacientes relatam que a satisfação inclui a boa comunicação, orientação, confiabilidade, dignidade, respeito ao atendimento rápido, bom acesso ${ }^{[13]}$. 
Quando questionados se o profissional que atendeu o paciente deu importância a ele, 92,5\% (50) afirmaram ser ótimo, 5,5\% (3) bom e 1,8\% (1) regular. De acordo estudos quando o profissional possui empatia, demonstram carinho, preocupação e amor no cuidado, transmitindo ao usuário um acolhimento e uma sensação de bem-estar, mesmo quando há uma superlotação no pronto socorro[18].

Sobre a privacidade preservada durante o atendimento, 96,2\% (51) relataram que foi ótimo, $1,8 \%$ (1) bom e $1,8 \%$ (1) regular. A privacidade é um direito do usuário, podendo ser realizada através de cortinas ou divisórias móveis, melhorando a satisfação, o atendimento e a interação da equipe ${ }^{[11]}$.

\subsection{VISÃO DA EQUIPE DE ENFERMAGEM SOBRE O SERVIÇO DE URGÊNCIA E EMERGÊNCIA}

Quando questionado aos técnicos de enfermagem se todos os pacientes são orientados antes da realização dos procedimentos, 50\% afirmaram ser ótimo, 33,3\% regular e $16,6 \%$ bom. Autores que realizaram um estudo com 382 participantes em uma unidade de urgência e emergência em Fortaleza, os resultados não foram satisfatórios na orientação dos profissionais para com os pacientes apontando que os mesmos não orientavam os pacientes quanto aos procedimentos ${ }^{[15]}$.

Sobre a separação dos usuários conforme classificação de risco, 66,6\% (4) afirmaram ser regular, 16,6\% (1) bom e 16,6\% (1) ótimo. O objetivo da classificação de risco, é avaliar a condição e separar os pacientes de acordo com a gravidade, os com risco de vida, com necessidade de atendimento imediato e os usuários que podem aguardar ${ }^{[19]}$.

Referente às orientações aos familiares e ao paciente a cada procedimento, houve uma concordância, 33,3\% afirmaram ser regular, 33,3\% bom e 33,3\% ótimo. Estudo realizado demonstrou a importância do acolhimento aos familiares, para suprir suas necessidades ${ }^{[3]}$.

Deve haver uma boa comunicação e explicação dos procedimentos a ser realizado, saber lidar com a família do paciente, que se encontram nas mesmas situações de medo é muito importante ${ }^{[20]}$.

A visão do enfermeiro sobre o conforto proporcionado aos pacientes pela estrutura física do setor, 33,3\% (1) consideram ruim, 33,3\% (1) regular, 33,3\% (1) bom. Um estudo sobre acolhimento e classificação de risco evidenciou a necessidade de melhoria no conforto ao usuário e ao acompanhante ${ }^{[21]}$.

Sobre a avaliação do espaço, se este proporciona privacidade ao usuário, todas as respostas dos profissionais de enfermagem mantiveram equilíbrio considerando o espaço, 33,3\% (1) péssimo, 33,3\% (1) ruim, 33,3\% (1) regular. 
Neto entrevistou 14 enfermeiros e concluiu que a estrutura física deixa a desejar, com isso há algumas fragilidades importantes, como por exemplo, a dificuldade do paciente em se sentir à vontade e expor sentimentos e o que está passando no momento[22].

Referente à comunicação entre os trabalhadores, há uma divergência nas respostas dos profissionais de enfermagem, sendo que $33,3 \%$ (1) relatou ser ruim, $33,3 \%$ (1) bom e 33,3\% (1) ótimo.

Estudo realizado com profissionais em um pronto socorro, na cidade de São Paulo e concluíram que, quando analisado a interação destes profissionais, o foco era somente o paciente, passando despercebida a comunicação entre os profissionais. A falta de comunicação dificulta o cuidado, acaba caindo na rotina e não atingem os objetivos totalmente ${ }^{[23]}$.

Em uma emergência, a comunicação é uma das principais atitudes a se tomar, deve-se transmitir calma e segurança, ouvindo atentamente e fornecendo todas as informações necessárias para o paciente, retirando todas as dúvidas e reduzindo a ansiedade que o mesmo se encontra ${ }^{[24]}$.

Quando questionados sobre a cobertura da classificação de risco com todos os pacientes atendidos no serviço de urgência e emergência $66,6 \%$ relataram ser ótimo e $33,3 \%$ regular.

De acordo com o Ministério da Saúde, a classificação de risco serve para humanizar o atendimento, diminuir o tempo de espera e determinar local de atendimento primário ${ }^{[14]}$.

Ao fazer uma auto avaliação sobre prestar uma assistência de forma acolhedora e resolutiva, 66,6\% (2) afirmaram ser bom e 33,3\% (1) ótimo.

Solla afirma a importância da assistência resolutiva, sendo parte do acolhimento. A escuta qualificada, a triagem bem feita, reduz a centralização de atendimentos e promove potencialidade aos profissionais ${ }^{[25]}$.

A visão do enfermeiro sobre a satisfação do usuário é que $66,6 \%$ (2) consideram ser regular e 33,3\% (1) bom. A satisfação inclui a boa comunicação, orientação, confiabilidade, dignidade, respeito ao atendimento rápido, bom acesso $^{[13]}$.

Quando questionados sobre a organização do serviço de acordo com a classificação de risco, 66,6\% (2) afirmaram ser bom e 33,3\% (1) ruim.

O Ministério da Saúde também afirma que, a classificação de risco organiza os pacientes não pela ordem de chegada, mas sim pela urgência do atendimento. Essa classificação é importante para informar o paciente do possível tempo de espera, informar quando não há um risco iminente, aumentar a satisfação do usuário, tornar a equipe mais organizada[26]. 


\section{CONCLUSÃO}

O estudo realizado foi de grande importância porque foram obtidas opiniões tanto dos pacientes quanto dos técnicos e enfermeiros, sendo de diferentes aspectos.

Os principais desafios para atuação em urgência e emergência hospitalar apontados são a necessidade de atender todos os pacientes de modo rápido e eficiente, realizar a classificação de risco corretamente, administrar o setor de forma organizada, a sobrecarga de trabalho, a superlotação de atendimentos, poucos profissionais em atendimento no setor, entre outros.

O tempo de espera de uma unidade de urgência e emergência só seria resolvido com a separação dos pacientes que deveriam ser atendidos no posto de saúde e os que realmente necessitam do pronto socorro, diminuindo o fluxo dos pacientes. O espaço para o acompanhante é difícil por envolver uma realização de uma estrutura diferenciada.

As pessoas doentes são bastante vulneráveis, o ambiente hospitalar é triste, infeliz, carregado, desanimado, pois trata do ser doente, portanto é necessário que haja um investimento profissional e pessoal no seu autocuidado. A ideia que se tem é de que quem cuida sabe se cuidar, então necessariamente, não precisa ser cuidado, porém, a realidade é diferente, as pessoas que cuidam precisam ser cuidadas porque pode causar desgaste físico e emocional.

Mesmo com os avanços, palestras e recursos de informações que temos hoje, ainda se torna necessário "frisar" a importância do acolhimento, de ter uma escuta qualificada e empatia perante o outro. Os profissionais possuem dificuldades em tratar o doente e não a doença.

Conclui-se que devem ser realizados diversos cursos profissionalizantes obrigatórios que proporcionem a visão da humanização, melhorando o atendimento e a qualidade de vida dos usuários.

Devido à complexidade da atuação, faz-se necessário explorar as características e habilidades dos profissionais que atuam no serviço de urgência e emergência, visando aperfeiçoar a atuação dos mesmos e também contribuir para direcionar a formação acadêmica. É necessário um trabalho humanizado, com uma equipe organizada e comunicativa, bem estruturada e atualizada, para melhor atendimento do paciente.

É importante salientar que o estudo traz novas possibilidades de pesquisas, considerando aspectos relacionados à saúde do trabalhador, necessidade de educação permanente, dimensionamento de pessoal entre outros, bem como aspectos relacionados aos pacientes e seus acompanhantes, para que com um diagnóstico situacional possam apontar estratégias de melhoria na qualidade e segurança do cuidado possibilitando o fortalecimento do SUS. 


\section{REFERÊNCIAS}

1. Angnes, D. I. Humaniza saúde: diferencial do atendimento na saúde. Porto Alegre, 2004. [Acesso em: 04 dez 2018.] Disponível em: file://C:/Users/user/Downloads/20140521105954v18n2_04humanizasaude.pdf

2. Jorge, V. C. et al. Equipe de enfermagem e detecção de indicadores de agravamento em pacientes de pronto-socorro. Maringá, 2012. [Acesso em: 02 dez 2018] Disponível em: https://www.redalyc.org/pdf/1277/127728365018.pdf

3. Garçon, T. A. et al. Fatores desencadeantes de estresse do enfermeiro na unidade de urgência e emergência. Revista Enfermagem Atual InDerme, v. 87, n. 25, 2019.

4. Motta, A. L. Auditoria de enfermagem no processo de credenciamento. São Paulo: látria, 2003.

5. Freire, G. V. et al. Liderança do enfermeiro nos serviços de urgencia e emergencia: revisão integrativa/Nursing leadership in urgency and emergency services: integrative review. Brazilian Journal of Health Review, v. 2, n. 3, p. 2029-2041, 2019.

6. Gomes, S. F.; Santos, M. M.; Carolino, E. T. Riscos psicossociais no trabalho: Estresse e estratégias de coping em enfermeiros em oncologia. Revista LatinoAmericana de Enfermagem, 21(6), 1282-1289. 2013.

7. Martins, J. T. et al. Significados de cargas de trabalho para enfermeiros de pronto socorro/emergência. Ciência, Cuidado e Saúde, 12(1), 40-46. 2013.

8. Santos, R. C., Miranda, F. A. The roles of the professional and the policies of mental health in the city of Natal, Brazil: An analytic study. Online Brazilian Journal of Nursing,13 (sup. 1), 475-477, 2014.

9. Albuquerque, M. C. dos S. de et al. Empatia dos profissionais de enfermagem de um serviço hospitalar de emergência. Texto \& Contexto-Enfermagem, v. 28, 2019.

10. Moura, J. et al. Nursing management and the conflict management in emergency care in hospital. J Nurs UFPE. 2011.

11. Morais, A. S.; Melleiro, M. M. A qualidade da assistência de enfermagem em uma unidade de emergência: a percepção do usuário. Rev. Eletr. Enf. [Internet]. jan/mar;15(1):112-20. 2013. [Acesso em: 25 jul. 2018] Disponível em: https://revistas.ufg.br/fen/article/view/15243/15528 
12. Mendes, A. da C. G. Avaliação da qualidade da assistência de urgência e emergência: uma abordagem por triangulação de métodos. Recife, 2009. [Acesso em: 25 jul 2018]. Disponível em:

http://www.cpqam.fiocruz.br/bibpdf/2009mendes-acg.pdf

13. Mishima, S. M. et al. Satisfação do usuário sob a perspectiva da responsividade: estratégia para análise de sistemas universais?. 2016. [Acesso em: 02 dez. 2018] Disponível em: http://www.scielo.br/pdf/rlae/v24/pt_01041169-rlae-02674.pdf

14. Brasil, Ministério da Saúde. As cartas de promoção da saúde. Brasília, 2002. [acesso em: 02 dez 2018]. Disponível em:

http://bvsms.saude.gov.br/bvs/publicacoes/cartas_promocao.pdf

15. Guedes, M. V. C.; Henriques, A. C. P. T.; Lima, M. M. N. Acolhimento em um serviço de emergência: percepção dos usuários, Brasília, 2013. [Acesso em: 02 dez 2018]. Disponível em http://www.scielo.br/pdf/reben/v66n1/v66n1a05.pdf

16. Nascimento, E. R. P. et al. Classificação de risco na emergência: avaliação da equipe de enfermagem. 2017. [acesso em: 29 jul. 2018]. Disponível em: http://www.facenf.uerj.br/v19n1/v19n1a14.pdf

17. Gomide, M. F. S.; Pinto, I. C.; Figueiredo, L. A. Acessibilidade e demanda em uma Unidade de Pronto Atendimento: perspectiva do usuário. São Paulo, 2012. [Acesso em: 02 dez. 2018]. Disponível em:

https://www.redalyc.org/html/3070/307026829008/

18. Bagglo, M. A.; Callegaro, G. D.; Erdmann, A. L. Compreendendo as dimensões de cuidado em uma unidade de emergência hospitalar. Florianópolis, 2008. [Acesso em: 02 dez. 2018]. Disponível em:

http://www.scielo.br/pdf/reben/v61n5/a04v61n5.pdf

19. Coutinho, A. A. P.; Cecilio, L. C. de O.; Mota, J. A. C. Classificação de risco em serviços de emergência: uma discussão da literatura sobre o Sistema de Triagem de Manchester. Minas Gerais, 2012. [Acesso em: 02 dez. 2018]. Disponível em: http://rmmg.org/artigo/detalhes/101

20. Grassi, M. de F. O.; Maria, M. A.; Quadros, F. A. A. Sistematização da assistência de enfermagem em serviços de urgência e emergência: viabilidade de implantação. Brasília, 2012. [Acesso em: 25 jun. 2018]. Disponível em: http://www.redalyc.org:9081/html/2670/267028449015/

21. Junior, J. A. B.; Matsuda, L. M. Construção e validação de instrumento para avaliação do acolhimento com classificação de risco. Maringá, 2012.

[Acesso em: 02 dez. 2018]. Disponível em:

https://www.redalyc.org/html/2670/267025266006/ 
22. Neto, A. V. de L. et al. Classificação de risco em emergência hospitalar: relações entre a prática, o profissional e o usuário, Rio Grande do Norte, 2016. [Acesso em: 02 dez. 2018]. Disponível em:

https://revistainterdisciplinar.uninovafapi.edu.br/index.php/revinter/article/view/89 3/pdf_306

23. Souza, R. B.; Silva, M. J. P.; Nori, A. Pronto-socorro: uma visão sobre a interação entre profissionais de enfermagem e pacientes. São Paulo, 2007. [Acesso em: 02 dez. 2018]. Disponível em:

file://C:/Users/user/Downloads/Pronto-

Socorro_uma_visao_sobre_a_interacao_entre_p\%20(1).pdf

24. Barbieri, R. L. S.O.S.: cuidados emergenciais. São Paulo: Rideel, 2002.

25. Solla, J. J. S. P. Acolhimento no sistema municipal de saúde. Brasília, 2005. [Acesso em: 02 dez. 2018]. Disponível em:

http://www.scielo.br/pdf/rbsmi/v5n4/27768.pdf

26. Brasil, Ministério da Saúde. Acolhimento e classificação de risco nos serviços de urgência. Ministério da Saúde, Secretaria de Atenção à Saúde, Política Nacional de Humanização da Atenção e Gestão do SUS. Brasília, 2009.

Artigo recebido em: 06/12/2018

Artigo aprovado em: 09/09/2019

Artigo publicado em: 16/09/2019 